\title{
Extreme ionization of heavy atoms in solid-density plasmas by relativistic second-harmonic laser
} pulses

\author{
R. Hollinger $\oplus^{1 凶}$, S. Wang1, Y. Wang', A. Moreau $\oplus^{1}$, M. G. Capeluto $\oplus^{1,2}$, H. Song ${ }^{1}{ }^{1}$, A. Rockwood ${ }^{3}$, \\ E. Bayarsaikhan ${ }^{3}$, V. Kaymak ${ }^{4}$, A. Pukhov ${ }^{4}{ }^{4}$, V. N. Shlyaptsev' and J. J. Rocca ${ }^{1,3 凶}$
}

\begin{abstract}
Stripping heavy atoms in solid matter of most of their electrons requires the extreme conditions that exist in astrophysical plasmas, but are difficult to create in the laboratory ${ }^{1-3}$. Here we demonstrate solid-density gold plasmas with atoms stripped of up to 72 electrons $\left(\mathrm{N}\right.$-like $\mathrm{Au}^{\mathbf{7 2}+}$ ) over large target depths. This record ionization is achieved by irradiating solid foils and near-solid-density nanowire arrays with highly relativistic $\left(3 \times 10^{21} \mathrm{~W} \mathrm{~cm}^{-2}\right)$ second-harmonic femtosecond laser pulses of $<10 \mathrm{~J}$ energy focused into a $1.6 \mu \mathrm{m}$ spot. The short wavelength and high intensity enable the interaction to occur at a relativistic critical density 4,5 of $10^{23} \mathrm{~cm}^{-3}$. Solid targets reach a higher average charge in 1- to 2- $\mu \mathrm{m}$-thick layers, while the less dense nanowire plasmas are heated to much larger depths $(>8 \mu \mathrm{m})$ by energetic electrons generated near the nanowire tips. Larger laser spots could result in solid Au plasmas ionized up to He-like.
\end{abstract}

Extending the accessible states of matter in the laboratory into extreme unexplored regimes can provide a new understanding of the behaviour of atoms in such environments, leading to the discovery of new phenomena and applications. The ability to generate data from solid-density plasmas with unprecedented degrees of ionization will provide much needed benchmarks to extend the capability of models to simulate ultrahigh energy density states of matter. For example, a comparison of high-resolution X-ray spectra with advanced atomic codes could lead to new insights into the behaviour of extremely charged atoms in intense micro-fields and also address new questions on the physics of these ions in solid-density environments. Au atoms at densities over 10 orders of magnitude below solid density have been ionized up to the Ne-like stage $\left(\mathrm{Au}^{69+}\right)$ by collisions with a high-energy electron beam while being trapped in a potential well, producing high-quality L-shell spectra over a broad range of high ion charges ${ }^{6,7}$. Achieving similar degrees of ionization in near-solid-density plasmas requires the deposition of ultrahigh energy densities, leading to extreme temperatures and high collisional ionization rates. High degrees of ionization have been achieved in dense plasmas driven by laser pulse energies of several hundred joules to kilojoules. Experiments conducted at the OMEGA laser using $9 \mathrm{~kJ}$ pulses of nanosecond duration to heat a reduced-scale 'hot' hohlraum revealed strong L-shell Au emission with an average charge state of Ti-like $\mathrm{Au}^{57+}$ and an inferred temperature of $6.5 \mathrm{keV}$ at an electron density of $10^{21} \mathrm{~cm}^{-3}$ (ref. ${ }^{3}$ ). For mid-atomic-number $(Z)$ elements such as $\mathrm{Ni}$, measurements performed at intensities up to $5 \times 10^{20} \mathrm{~W} \mathrm{~cm}^{-2}$ with $400 \mathrm{~J}$ pulses of $0.8 \mathrm{ps}$ duration revealed emission from the $\mathrm{He}-\alpha$ and $\mathrm{Ly}-\alpha$ transitions and a surface temperature of $5 \mathrm{keV}$ decreasing to $600 \mathrm{eV}$ at a depth of $1.3 \mu \mathrm{m}$ (ref. ${ }^{8}$ ). Temperatures of $600 \mathrm{eV}$ in $\mathrm{Al}$ foils buried under $10 \mu \mathrm{m}$ of $\mathrm{CH}$ were measured by $\mathrm{K}$-shell emission spectroscopy when irradiated by $100 \mathrm{~J}$ pulses of 0.5 ps duration $^{9}$. Buried $\mathrm{Cu}$ tracers irradiated at an intensity of $>10^{20} \mathrm{~W} \mathrm{~cm}^{-2}(140 \mathrm{~J}, 1.053 \mu \mathrm{m}, 500 \mathrm{fs}$ duration) at the Titan short pulse laser exhibited a large amount of He-like emission with an estimated surface temperature of $2,100 \mathrm{eV}$ $\left(\right.$ ref. $\left.{ }^{10}\right)$. Experiments irradiating $12 \%$ solid-density $\mathrm{Au}$ nanowire arrays at an intensity of $5 \times 10^{18} \mathrm{~W} \mathrm{~cm}^{-3}$ generated plasmas ionized to charge states up to $\mathrm{Au}^{52+}$ (ref. ${ }^{11}$ ).

Here we demonstrate the generation of solid- and near-solid-density Au plasmas characterized by L-shell line emission from atoms ionized up to the $\mathrm{N}$-like state $\mathrm{Au}^{72+}$, an unprecedented degree of ionization for dense laboratory plasmas. Moreover, this was achieved using femtosecond laser pulses of less than $10 \mathrm{~J}$ energy, which can be generated at high repetition rates. The experimental results were obtained using high-contrast second-harmonic $\lambda=400 \mathrm{~nm}$ laser pulses focused to a highly relativistic intensity of $\sim 3 \times 10^{21} \mathrm{~W} \mathrm{~cm}^{-2}$, corresponding to a normalized vector potential of the laser field of $a_{0}=18.6$. At this intensity, relativistic effects increase the critical electron density $\left(n_{\text {crit }}\right)$ by a factor of $\gamma \approx 13$ to $n_{\text {crit rel }} \approx 10^{23} \mathrm{~cm}^{-3}\left(n_{\text {crit rel }}=\gamma n_{\text {crit }}\right.$; relativistic factor $\left.\gamma=\sqrt{1+\frac{a_{o}^{2}}{2}}\right)$. This increase in the critical density makes it is possible to volumetrically deposit energy generating highly ionized atoms deeper into overdense media. We compare results from solid slab targets to those obtained when irradiating nanowire arrays, which previous studies at lower intensities have shown to result in enhanced X-ray emis$\operatorname{sion}^{12-16}$. We show that, at these highly relativistic intensities, it is possible to volumetrically heat solid-density plasmas to depths of $>1 \mu \mathrm{m}$ (with an estimated energy deposition density of up to $10 \mathrm{GJ} \mathrm{cm}^{-3}$ ) and aligned nanowire arrays with $15 \%$ solid density to depths $>8 \mu \mathrm{m}$. For nanowires, the average volumetric energy density approaches $100 \mathrm{GJ} \mathrm{cm}^{-3}$, an ultrahigh energy density regime that is virtually unexplored in laboratory plasmas. Three-dimensional (3D) particle-in-cell (PIC) simulations show that the mechanism of energy deposition deep into the nanowire target differs from those at lower irradiation intensity and is dominated by energetic electrons accelerated near the target surface.

The experiments were conducted by irradiating gold foils and nanowire arrays with a frequency-doubled Ti:sapphire laser ${ }^{17}$ delivering ultrahigh-contrast $\left(>10^{12}\right), \lambda=400 \mathrm{~nm}$ pulses of $45 \mathrm{fs}$ duration

'Electrical and Computer Engineering Department, Colorado State University, Fort Collins, CO, USA. 'Departamento de Física, Universidad de Buenos Aires - IFIBA, Ciudad Universitaria, Buenos Aires, Argentina. ${ }^{3}$ Physics Department, Colorado State University, Fort Collins, CO, USA. ${ }^{4}$ Institut für Theoretische Physik, Heinrich-Heine-Universität Düsseldorf, Düsseldorf, Germany.凶e-mail: reed.hollinger@colostate.edu; jorge.rocca@colostate.edu 


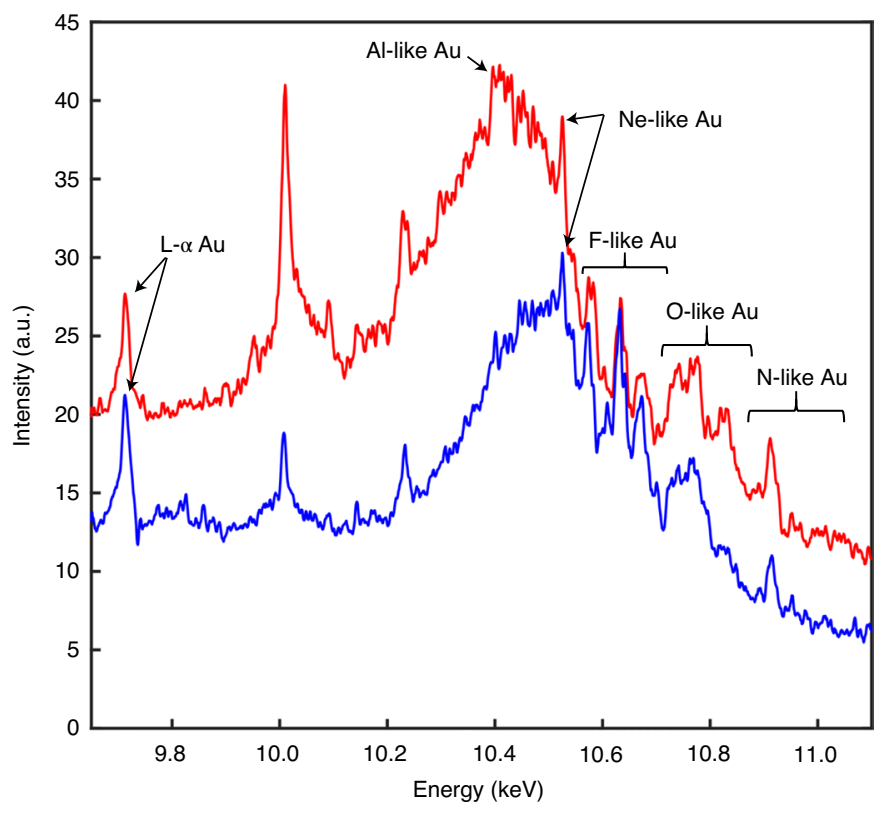

Fig. 1 | L-shell emission from solid-density Au foils and Au nanowire arrays. L-shell emission from solid-density Au foils (blue curve) and 100-nm-diameter Au nanowire arrays at $15 \%$ of solid density (red curve) irradiated at $3 \times 10^{21} \mathrm{~W} \mathrm{~cm}^{-2}$. Line emission from charge states including $\mathrm{Ne}$-like $\mathrm{Au}^{69+}, \mathrm{F}$-like $\mathrm{Au}^{70+}, \mathrm{O}$-like $\mathrm{Au}^{71+}$ and $\mathrm{N}$-like $\mathrm{Au}^{72+}$ is observed for both targets. The line feature near $10.92 \mathrm{keV}$ has contributions of $\mathrm{N}$-like $\mathrm{Au}^{72+}$ and $\mathrm{O}-$ like $\mathrm{Au}^{71+}$. The charge state distribution is centred around Al-like $\mathrm{Au}^{66+}$ for the nanowire plasma and $\mathrm{Ne}-$ like $\mathrm{Au}^{69+}$ for the solid foil plasma.

on target. The use of second harmonics suppresses laser pre-pulses and leads to higher critical densities and, consequently, increased collisional rates. The L-shell emission of Au was monitored using a moderate-resolution $(E / \Delta E \approx 1,000)$ Von Hamos mica crystal spectrometer. Figure 1 shows spectra corresponding to both a Au foil target and a $100-\mathrm{nm}$-diameter Au nanowire array with $15 \%$ of solid density. Both targets display emission from the Ne-like Au transition, at $10,525 \mathrm{eV}$ as well as lines corresponding to higher ionization stages up to $\mathrm{N}$-like $\mathrm{Au}^{72+}$. The average charge state is measured to be Al-like $\mathrm{Au}^{66+}$ for nanowires and at Ne-like $\mathrm{Au}^{69+}$ for solid targets, which is substantially higher than reported in previous work. Simulated spectra computed with the hydrodynamic/atomic physics code RADEX using initial conditions from the PIC simulations agree with the experiment in showing line emission from ions up to $\mathrm{N}$-like $\mathrm{Au}^{72+}$ (Extended Data Fig. 1).

The energy penetration depth at this intensity was measured by monitoring the $\mathrm{He}-\alpha$ line emission (Fig. $2 \mathrm{a}-\mathrm{d}$ ) from a buried $\mathrm{Ni}$ tracer under a variable amount of solid Au (inset, Fig. 2e). Figure $2 \mathrm{c}$ shows that He-like $\mathrm{Ni}$ line emission is still visible when the Ni tracer is under $1 \mu \mathrm{m}$ of solid Au. A summary of the He-like Ni emission intensity versus buried depth is plotted in Fig. 2e. The much larger laser heat penetration in the nanowire arrays was demonstrated using an array of dual-composition 100-nm-diameter wires with $15 \%$ of solid density consisting of an $8-\mu \mathrm{m}$ Au nanowire segment on top of a $3 \mu \mathrm{m}$ Ni segment. The He-like Ni lines are still visible under $8 \mu \mathrm{m}$ of $\mathrm{Au}$, revealing that the nanowire plasmas are significantly larger in volume than the solid-density target plasmas. This was simultaneously measured to contribute to an increase in the $h v>1 \mathrm{keV} \mathrm{X}$-ray emission with respect to the foil targets (Extended Data Fig. 2) due to the increase in hydrodynamic cooling time compared with the plasma radiative lifetime.
PIC simulations show that, at these highly relativistic intensities, the mechanism by which energy is deposited deep into the dense array of small-diameter nanowires differs from that at lower intensities, where laser light penetrates deep into the interwire gaps $^{11}$. Figure 3 shows the results for a $15 \%$ solid-density array of 100-nm-diameter Au nanowires. The electron density in units of $n_{\text {crit }}=6.9 \times 10^{21} \mathrm{~cm}^{-3}$ (Fig. $3 \mathrm{a}-\mathrm{c}$ ), the electric field propagation (Fig. $3 \mathrm{~d}-\mathrm{f}$ ) and the ionization state (Fig. $3 \mathrm{~g}-\mathrm{i}$ ) are shown for three different times: $-30 \mathrm{fs}, 0 \mathrm{fs}$ and $+1,000 \mathrm{fs}$ with respect to the peak of the laser pulse. Initially, the electric field of the laser propagates in the interwire gap down the length of the wire, accelerating electrons towards the substrate (Fig. 3a). As the laser intensity increases, a large electric field at the nanowire tips drives optical field ionization up to $\mathrm{Ne}$-like $\mathrm{Au}^{69+}$ and accelerates the electrons towards the substrate (Fig. $3 \mathrm{~h}$ ). The nanowires begin to pinch due to a quasi-static self-generated magnetic field generated by the return electron current ${ }^{18}$ through the wires, and the peak electron density within the wires exceeds $1,000 n_{\text {crit }}$ (Fig. 3e). The wire tips are rapidly heated by electron collisions and explode before the peak of the laser pulse arrives (Fig. 3b), filling the gaps with an overdense plasma greater than 15 times the critical density, which is higher than even the relativistic critical density. After this time, the laser can no longer propagate into the nanowire array. However, the laser heats up the thin surface layer, depositing a majority of the energy into a thin slab of overdense plasma, which reaches a peak electron energy density of $>1,000 \mathrm{GJ} \mathrm{cm}^{-3}$ (Extended Data Fig. 3). Energetic electrons emanating from this layer propagate down the length of the wires, depositing their energy into the array and ionizing atoms up to the $\mathrm{N}$-like stage in the entire volume. The average degree of ionization, Al-like $\mathrm{Au}^{66+}$, is lower than in the solid-density plasmas, but the plasma depth is nearly an order of magnitude higher, in agreement with the experimental observations.

In contrast to the nanowire target, the solid-density target volume is overdense for the entire duration of the laser pulse. The laser energy is absorbed mainly by collisionless processes ${ }^{19}$ with electrons and ions in a layer that is much thinner than the nanowire-array skin depth, causing less efficient energy absorption. A large population of energetic electrons generated within the skin depth transport the energy deep into the solid-density target, where current filaments give rise to Weibel instabilities ${ }^{20}$ (Extended Data Fig. 4). In the 3D PIC simulation for solid-density Au slab targets presented in Fig. 4 , the ionization of atoms to high charge states at $+500 \mathrm{fs}$ after the peak of the laser pulse is computed to propagate $\sim 2 \mu \mathrm{m}$ into the target, a value similar to that inferred from spectral measurements. This is in contrast to solid-density foils irradiated at much lower intensity, where only a thin layer is heated ${ }^{21}$. This larger depth of heated solid-density plasma increases the hydrodynamic expansion time, resulting in a new volumetrically heated solid-density plasma regime with an extreme degree of ionization. At non-relativistic intensities, the second or third harmonic of optical lasers is usually used to increase the absorption in flat targets at normal incidence. By comparison, the combination of the second harmonic with the relativistically modified critical density increases $n_{\text {crit }}$ to $\sim 10^{23} \mathrm{~cm}^{-3}$, corresponding to that of the 10th harmonic of a $1 \mu \mathrm{m}$ laser, effectively increasing absorption. Although the overall absorption of flat solid targets is still smaller than for nanowires, its increase, along with the increase in collisional rate, allows solid targets to reach and even surpass the average $Z$ in nanowires. Instead, it has been shown previously that, at lower intensities, solid targets generate much lower temperatures and lower ionization degrees than nanowires. The study of the plasma dynamics predicted by the PIC simulation will require ultrafast probes. For example, the intense femtosecond pulses from X-ray free-electron lasers will allow for Thomson scattering measurements of the plasma parameters once petawatt-class lasers capable of generating the plasmas reported here are implemented at those facilities. 

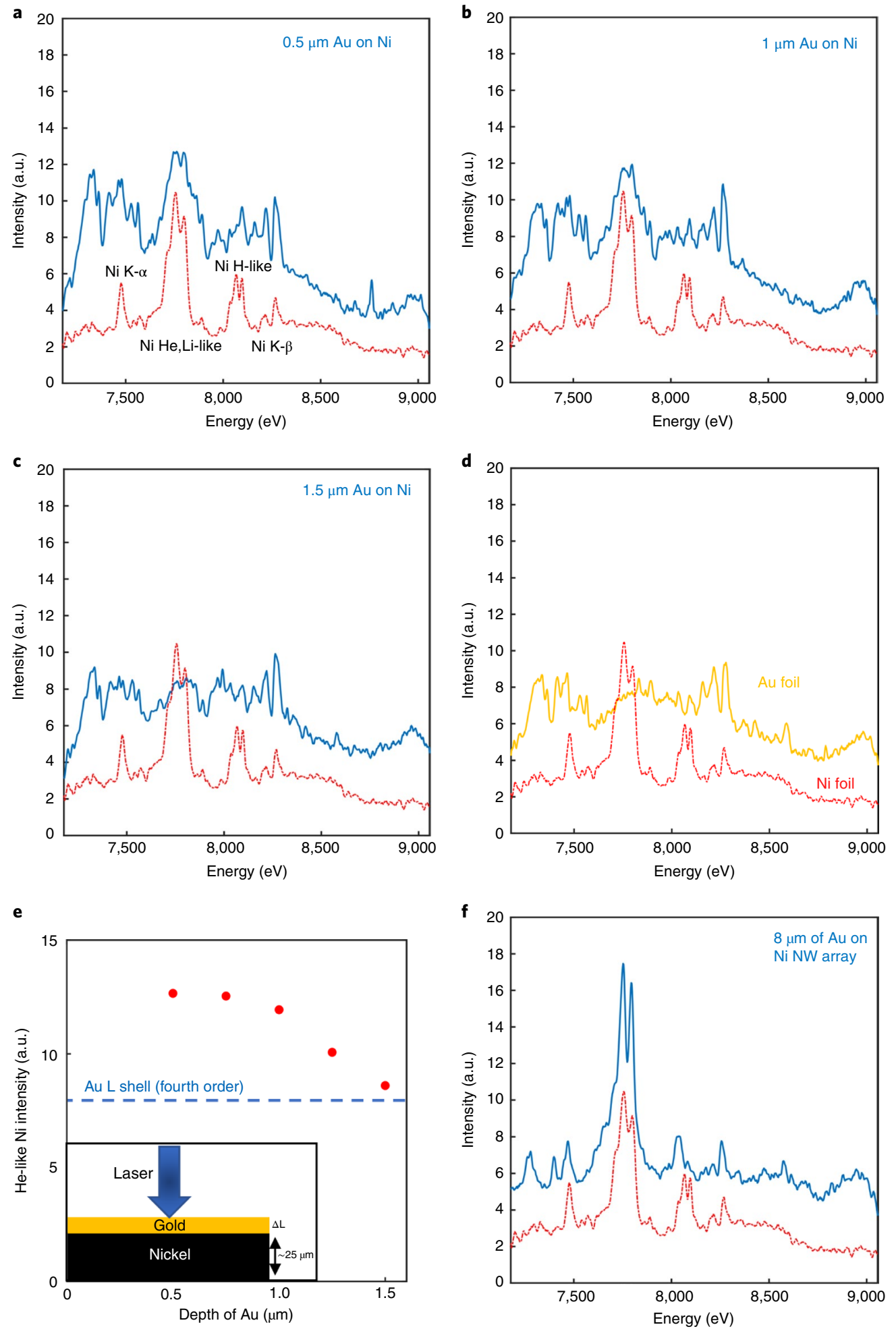

Fig. 2 | Energy penetration depth determined with buried layer spectroscopy. a-d, Single-shot spectra of Ni tracer (blue) buried under varying amounts of gold $(0.5 \mu \mathrm{m}(\mathbf{a}) ; 1 \mu \mathrm{m}(\mathbf{b})$ and $1.5 \mu \mathrm{m}(\mathbf{c}))$ compared with the spectra of a pure Au foil (d). Pure Ni foil reference spectra are plotted in red in each plot. e, Integrated $\mathrm{He}$-like $\mathrm{Ni}$ intensity versus Au thickness. Inset: structure of the targets used in the tracer experiment. $\mathbf{f}$, Spectra showing the much deeper heat penetration depth in the nanowire (NW) array, corresponding to segmented 100-nm-diameter Au nanowires that are $8 \mu \mathrm{m}$ in length with an average density corresponding to $15 \%$ solid on top of $3 \mu \mathrm{m}$ of Ni nanowires.

Simulations also predict that irradiation of solid targets with the same intensity over a larger spot using the upcoming new generation of multi-petawatt lasers will create solid-density plasmas with atoms ionized up to He-like Au (Extended Data Fig. 5) over even larger penetration depths. This will result from the reduced plasma expansion rate associated with the larger plasma volume maintaining an extremely hot plasma with a density close to the relativistic critical density, for a longer time. The minimum spot size 


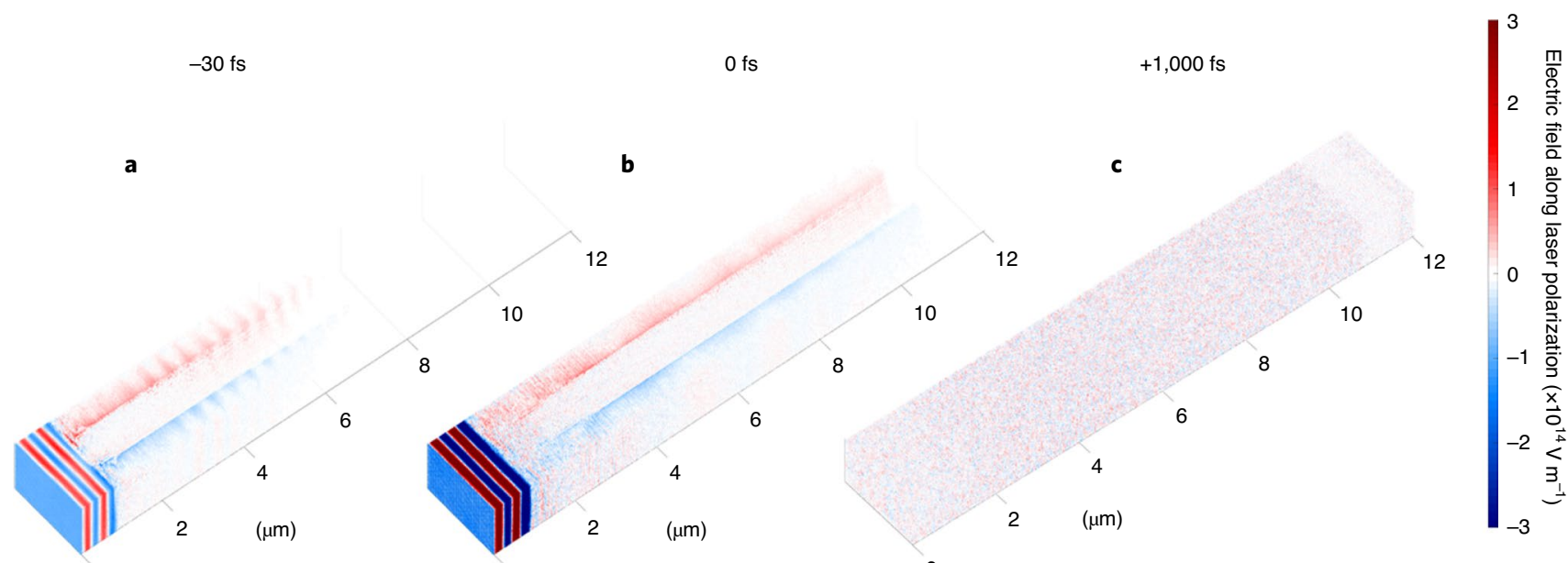

d

e

f

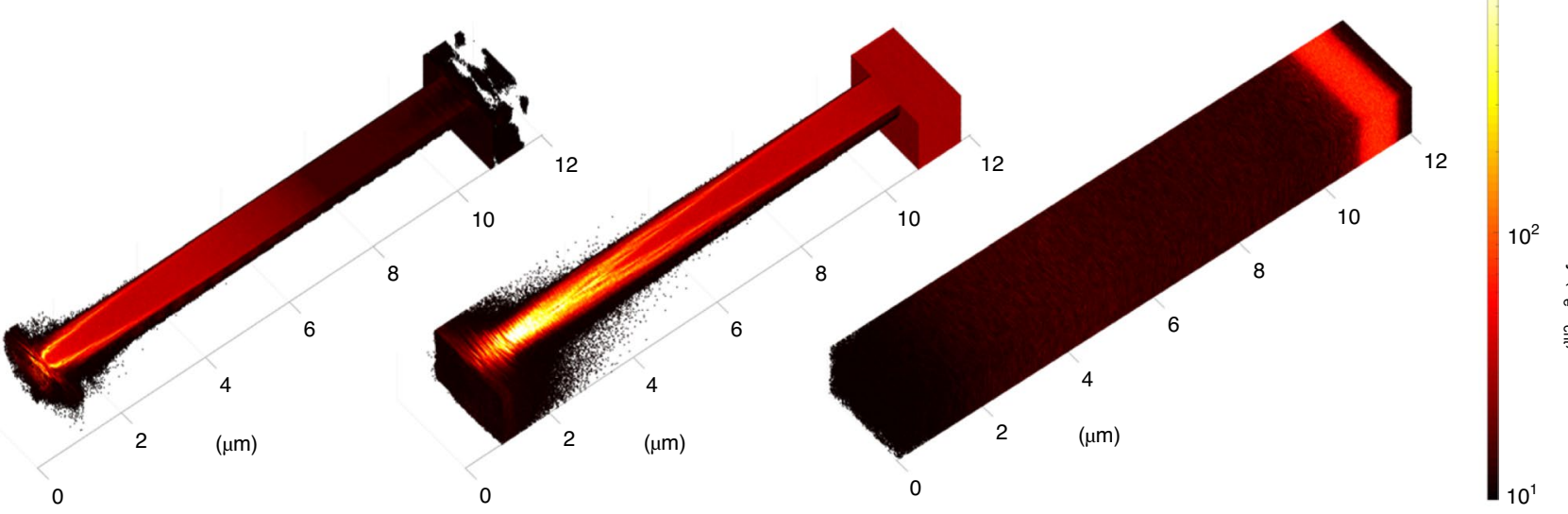

g

h

i

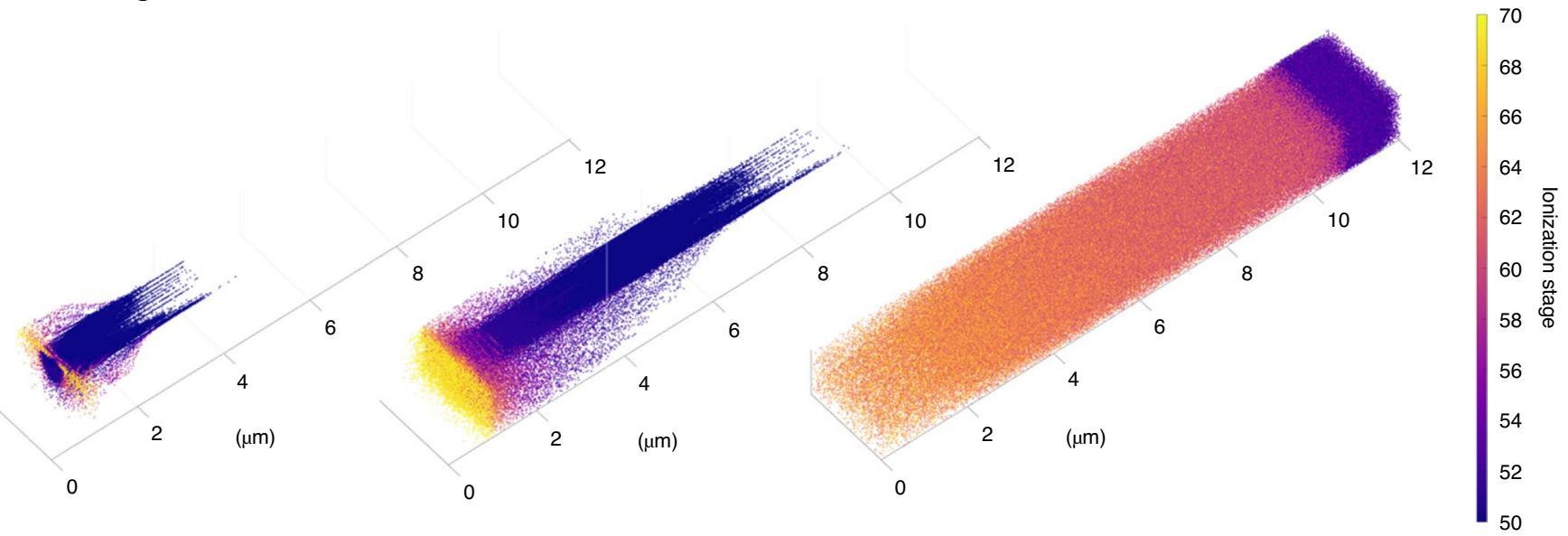

Fig. 3 | PIC simulation results for a 15\% solid-density array of 100-nm-diameter Au nanowires. a-i, 3D PIC simulations for an array of 100-nm-diameter Au nanowires with an average density corresponding to $15 \%$ solid density showing the evolution of the driving electric field (a-c), electron density (d-f) and ionization state $(\mathbf{g}-\mathbf{i})$ at three different times: $30 \mathrm{fs}$ preceding the peak of the laser pulse $(\mathbf{a}, \mathbf{d}, \mathbf{g})$, at the peak of the laser pulse $(\mathbf{b}, \mathbf{e}, \mathbf{h})$ and $+1,000 \mathrm{fs}$ afterwards $(\mathbf{c}, \mathbf{f}, \mathbf{i})$. The electron density is plotted in units of $n_{\text {crit }}\left(6.87 \times 10^{21} \mathrm{~cm}^{-3}\right.$ for $\left.\lambda=400 \mathrm{~nm}\right)$ and only for densities $>15 n_{\text {crit. }}$.

necessary to reach the He-like ionization state is estimated to be $\sim 5 \mu \mathrm{m}$. These unprecedented degrees of ionization in solid-density and near-solid-density nanostructure plasmas will open the door to the study of the atomic physics of highly charged atoms in very high-density plasmas, including, for example, phenomena such as line broadening, line shifts and continuum lowering. These unique plasmas will also enable applications such as beams of highly charged particles and more efficient X-ray radiation sources.

\section{Online content}

Any methods, additional references, Nature Research reporting summaries, source data, extended data, supplementary information, 


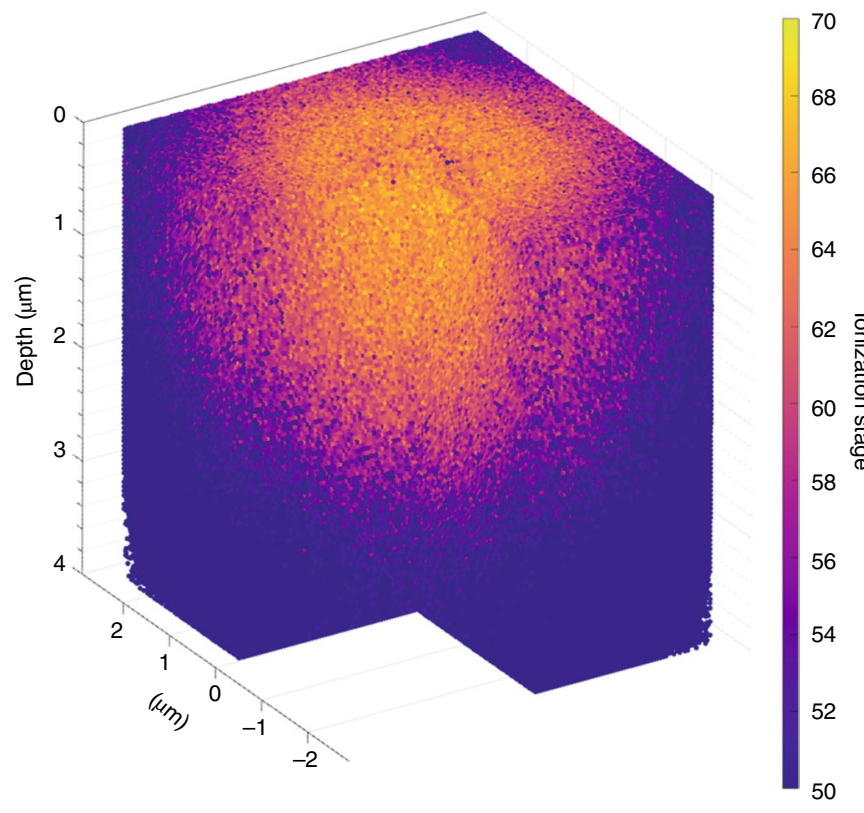

Fig. 4 | Three-dimensional PIC simulation showing the degree of ionization for a solid-density Au slab target. The target is irradiated at an intensity of $4 \times 10^{21} \mathrm{~W} \mathrm{~cm}^{-2},+500 \mathrm{fs}$ following the peak of the laser pulse.

acknowledgements, peer review information; details of author contributions and competing interests; and statements of data and code availability are available at https://doi.org/10.1038/ s41566-020-0666-1.

Received: 17 December 2019; Accepted: 18 June 2020;

Published online: 20 July 2020

\section{References}

1. Heeter, R. F. et al. Benchmark measurements of the ionization balance of non-local thermodynamic-equilibrium gold plasmas. Phys. Rev. Lett. 99, 195001 (2007).

2. Foord, M. E. et al. Ionization processes and charge-state distribution in a highly ionized high-Z laser-produced plasma. Phys. Rev. Lett. 85, 992-995 (2000).
3. May, M. J. et al. X-ray spectral measurements and collisional-radiative modeling of hot, gold plasmas at the omega laser. High Energy Density Phys. 4, 78-87 (2008).

4. Kaw, P. \& Dawson, J. Relativistic nonlinear propagation of laser beams in cold overdense plasmas. Phys. Fluids 13, 472-481 (1970).

5. Palaniyappan, S. et al. Dynamics of relativistic transparency and optical shuttering in expanding overdense plasmas. Nat. Phys. 8, 763-769 (2012).

6. Träbert, E. et al. L-shell spectroscopy of $\mathrm{Au}$ as a temperature diagnostic tool. Rev. Sci. Instrum. 79, 10E313 (2008).

7. Brown, G. V. et al. Investigation of the $2 p_{3 / 2}-3 d_{5 / 2}$ line emission of $\mathrm{Au}^{53+}-\mathrm{Au}^{69+}$ for diagnosing high energy density plasmas. Phys. Rev. E 77, 066406 (2008).

8. Akli, K. U. et al. Laser heating of solid matter by light-pressure-driven shocks at ultrarelativistic intensities. Phys. Rev. Lett. 100, 165002 (2008).

9. Hoarty, D. J. et al. Observations of the effect of ionization-potential depression in hot dense plasma. Phys. Rev. Lett. 110, 265003 (2013).

10. Chen, S. N. et al. X-ray spectroscopy of buried layer foils irradiated at laser intensities in excess of $10^{20} \mathrm{~W} / \mathrm{cm}^{2}$. Phys. Plasmas 16, 062701 (2009).

11. Purvis, M. A. et al. Relativistic plasma nanophotonics for ultrahigh energy density physics. Nat. Photon 7, 796-800 (2013).

12. Kulcsar, G. et al. Intense picosecond X-ray pulses from laser plasmas by use of nanostructured 'Velvet' targets. Phys. Rev. Lett. 84, 5149-5152 (2000).

13. Nishikawa, T. et al. Nanocylinder-array structure greatly increases the soft X-ray intensity generated from femtosecond-laser-produced plasma. Appl. Phys. B 73, 185-188 (2001).

14. Ovchinnikov, A. V. et al. Characteristic X-rays generation under the action of femtosecond laser pulses on nano-structured targets. Laser Part. Beams 29, 249-254 (2011)

15. Mondal, S. et al. Highly enhanced hard X-ray emission from oriented metal nanorod arrays excited by intense femtosecond laser pulses. Phys. Rev. B 83, 035408 (2011)

16. Hollinger, R. et al. Efficient picosecond X-ray pulse generation from plasmas in the radiation dominated regime. Optica 4, 1344-1349 (2017).

17. Wang, Y. et al. $0.85 \mathrm{PW}$ laser operation at $3.3 \mathrm{~Hz}$ and second-harmonic beamline. Opt. Lett. 42, 3828-3831 (2017).

18. Kaymak, V., Pukhov, A., Shlyaptsev, V. N. \& Rocca, J. J. Nanoscale ultradense Z-pinch formation from laser-irradiated nanowire arrays. Phys. Rev. Lett. 117, 035004 (2016).

19. Levy, M. C., Wilks, S. C., Tabak, M., Libby, S. B. \& Baring, M. G. Petawatt laser absorption bounded. Nat. Commun. 5, 4149 (2014).

20. Weibel, E. S. Spontaneously growing transverse waves in a plasma due to an anisotropic velocity distribution. Phys. Rev. Lett. 2, 83-84 (1959).

21. Rosmej, O. N. et al. Generation of keV hot near-solid density plasma states at high contrast laser-matter interaction. Phys. Plasmas 25, 083103 (2018).

Publisher's note Springer Nature remains neutral with regard to jurisdictional claims in published maps and institutional affiliations.

(c) The Author(s), under exclusive licence to Springer Nature Limited 2020 


\section{Methods}

Experimental. The experiments were conducted using laser pulses from the petawatt-class ALEPH Ti:sapphire laser developed at Colorado State University. The pulses were compressed to $45 \mathrm{fs}$ and were subsequently frequency-doubled using an 800 - $\mu \mathrm{m}$-thick type 1 second-harmonic-generation potassium diphosphate crystal. A contrast $>10^{12}$ is obtained after the second harmonic is separated from the fundamental using a series of five dichroic mirrors. A deformable mirror was used before the compressor to correct static aberrations in the wave front. The pulses were focused by an $f / 2,45^{\circ}$ off-axis parabola to achieve an intensity of up to $3 \times 10^{21} \mathrm{~W} \mathrm{~cm}^{-2}$ on target. Single-shot X-ray spectroscopy was performed using a mica crystal $(2 d=19.84 \AA)$ with a radius of curvature of $40 \mathrm{~mm}$ in a Von Hamos configuration in the third order. The spectrographs were recorded on a Fujifilm BAS MS image plate and read out immediately after every shot to avoid fading. The spectra are single shot. The experiment has good reproducibility (for example, see Extended Data Fig. 6 for three consecutive single-shot spectra obtained under the same irradiation conditions). Measurement of the energy penetration depth into the target was conducted by monitoring the ionized $\mathrm{K}$ shell of a buried Ni layer seen in the third order of a mica crystal. The $\mathrm{L}$ shell of $\mathrm{Au}$ is also present from the fourth order of the mica crystal, which has a significantly lower integrated reflectivity $^{22}$. The L-shell spectra were calibrated using the well-resolved He-like and $\mathrm{H}$-like transitions from the $\mathrm{K}$ shell of $\mathrm{Ge}$ at 10,274 eV and 10,569 eV (Extended Data Fig. 7). The X-ray yield was simultaneously measured using a filtered $\mathrm{Si}$ photodiode (AXUV100-G, International Radiation Detectors). A photodiode with a $13-\mu \mathrm{m}$-thick Be filter was placed $2.5 \mathrm{~m}$ from the plasma at $45^{\circ}$ with respect to the target normal to monitor the $h v>1 \mathrm{keV} \mathrm{X-ray} \mathrm{emission.} \mathrm{Large} \mathrm{surface} \mathrm{areas} \mathrm{of}$ high-aspect-ratio, aligned nanowires were fabricated through electrodeposition of $\mathrm{Ni}$ and $\mathrm{Au}$ into anodic aluminium oxide wafers.

PIC simulations. Both the solid-density and nanowire-array plasmas were simulated with a relativistic 3D PIC code Virtual Laser Plasma Laboratory ${ }^{23}$. This code is the same as that used to perform nanowire plasma simulations in ref. ${ }^{11}$, with atomic data added for high- $Z$ elements. The spectra were simulated using the radiative/hydrodynamic code $\mathrm{RADEX}^{24}$, with detailed atomic physics using data from the HULLAC code ${ }^{25}$. The PIC code standard algorithms were extended by packages for optical field ionization (OFI) and binary collisions, including electron impact ionization. OFI was treated as an under barrier tunnelling phenomenon in the static electric field ${ }^{26,27}$, with only sequential field ionization considered. The probabilities for Coulomb collisions between all particles in one mesh cell were calculated by a binary collision package. PIC simulations utilized a 3D geometry and self-consistently included ionization physics. A linearly polarized plane wave with $\lambda=400 \mathrm{~nm}$ and temporal Gaussian pulse profile with a duration of $45 \mathrm{fs}$ full-width at half-maximum (FWHM) was used to simulate the laser pulse with the normalized vector potential $a_{\mathrm{o}}=e A /\left(m c^{2}\right)=21$ for a laser intensity of $4 \times 10^{21} \mathrm{~W} \mathrm{~cm}^{-2}$. The laser impinged on the nanowire array and solid-density target at normal incidence. The PIC simulation space consisted of a cell volume encompassing the wire and interwire gaps, as well as space above the target to allow for expansion as it thermalized.

\section{Data availability}

The data that support the plots within this paper and other findings of this study are available from the corresponding authors upon reasonable request.

\section{Code availability}

The computer programs that support the findings of this study can be made available upon reasonable request to the corresponding authors.

\section{References}

22. Sánchez del Río, M., Faenov, A. Y., Pikuz, T. A., Souvorov, A. \& Freund, A. K Hard X-ray reflectivity of spherically bent mica crystals. AIP Conf. Proc. 521, 287-2292 (2000).

23. Pukhov, A. \& Meyer-ter-Vehn, J. Laser hole boring into overdense plasma and relativistic electron currents for fast ignition of ICF targets. Phys. Rev. Lett. 79, 2686-2689 (1997).

24. Shlyaptsev, V. N., Rocca, J. J. G. \& Osterheld, A. L. Dynamics of a capillary discharge X-ray laser. Proc. SPIE 2520, 365-372 (1995).

25. Bar-Shalom, A., Klapisch, M. \& Oreg, J. HULLAC, an integrated computer package for atomic processes in plasmas. J. Quant. Spectrosc. Radiat. Transf. 71, 169-188 (2001).

26. Karmakar, A. \& Pukhov, A. Collimated attosecond GeV electron bunches from ionization of high- $Z$ material by radially polarized ultra-relativistic laser pulses. Laser Part. Beams 25, 371-377 (2007).

27. Zhidkov, A. \& Sasaki, A. Effect of field ionization on interaction of an intense subpicosecond laser pulse with foils. Phys. Plasmas 7, 1341-1344 (2000).

\section{Acknowledgements}

The experiments were conducted using the Colorado State University ALEPH laser facility supported by LaserNet US (DE-SC0019076). The work was supported by Fusion Energy Sciences (FES), Office of Science, of the US Department of Energy (DOE) (grant no. DE-SC0014610). R.H. was supported by the US DOE FES Postdoctoral Research Program administered by the Oak Ridge Institute for Science and Education (ORISE) for the DOE. ORISE is managed by Oak Ridge Associated Universities (ORAU) under DOE contract number DE-SC0014664. All opinions expressed in this paper are the author's and do not necessarily reflect the policies and views of DOE, ORAU or ORISE. The simulations were conducted using the Colorado University/Colorado State University Summit High Performance Computer System, National Science Foundation (ACI1532235). We acknowledge the assistance of T. Kaiser in running the PIC code.

\section{Author contributions}

The concept of the experiment was proposed by R.H. and J.J.R. and the experiment was designed by R.H., Y.W., S.W. and J.J.R. Modelling was performed by V.N.S., R.H., V.K. and A.P. The experiments were conducted by R.H., A.M., S.W., Y.W., H.S. and A.R. The targets were developed and manufactured by M.G.C., E.B. and R.H. All authors participated in the writing of the manuscript.

\section{Competing interests}

The authors declare no competing interests.

\section{Additional information}

Extended data is available for this paper at https://doi.org/10.1038/s41566-020-0666-1. Correspondence and requests for materials should be addressed to R.H. or J.J.R. Reprints and permissions information is available at www.nature.com/reprints. 


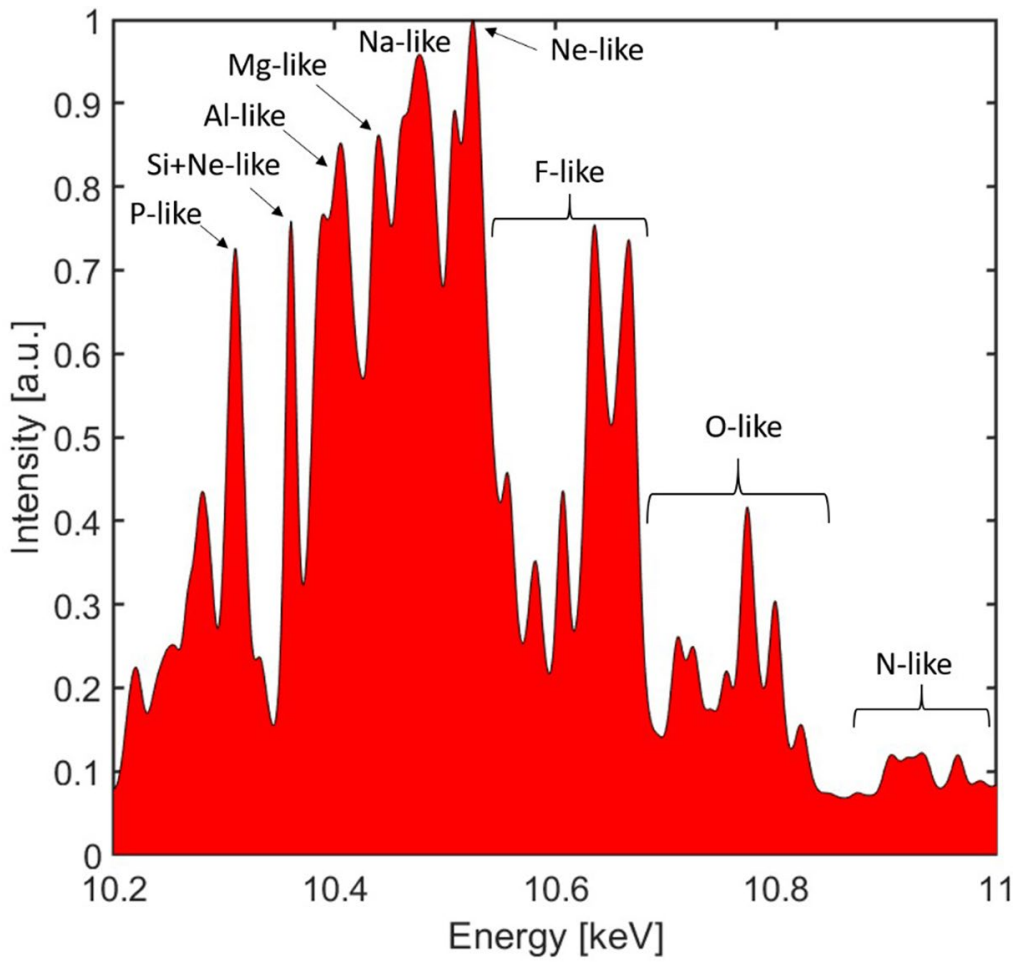

Extended Data Fig. 1 | Simulated Au spectrum. Simulated Au spectrum corresponding to solid flat gold target irradiated at an intensity of $4 \times 10^{21}$ Wcm ${ }^{-2}$. Best agreement with the experimental spectrum is obtained when lines from F-like to $\mathrm{N}$-like Au are shifted down in energy by $\sim 0.4 \%$. Similar shifts were necessary in ref. ${ }^{7}$ when comparing the calculated Au line energies with the measurement. The spectrum was computed using transient calculations performed with the atomic physics/hydrodynamic code RADEX using initial plasma parameters computed by the PIC simulations. The RADEX code uses detailed atomic data from the Hebrew University Lawrence Livermore Atomic Code (HULLAC) (ref. ${ }^{25}$ ). It was run using 200 to 1500 levels per ion from $\mathrm{Cu}$-like $\left(\mathrm{Au}^{50+}\right)$ to $\mathrm{B}$-like $\left(\mathrm{Au}^{74+}\right)$ to accurately simulate the large number of transitions from the $\mathrm{L}$ shell configuration. The simulated spectrum shows emission of all the observed lines for charge states from $\mathrm{Ne}$-like to $\mathrm{N}$-like $\mathrm{Au}$. 


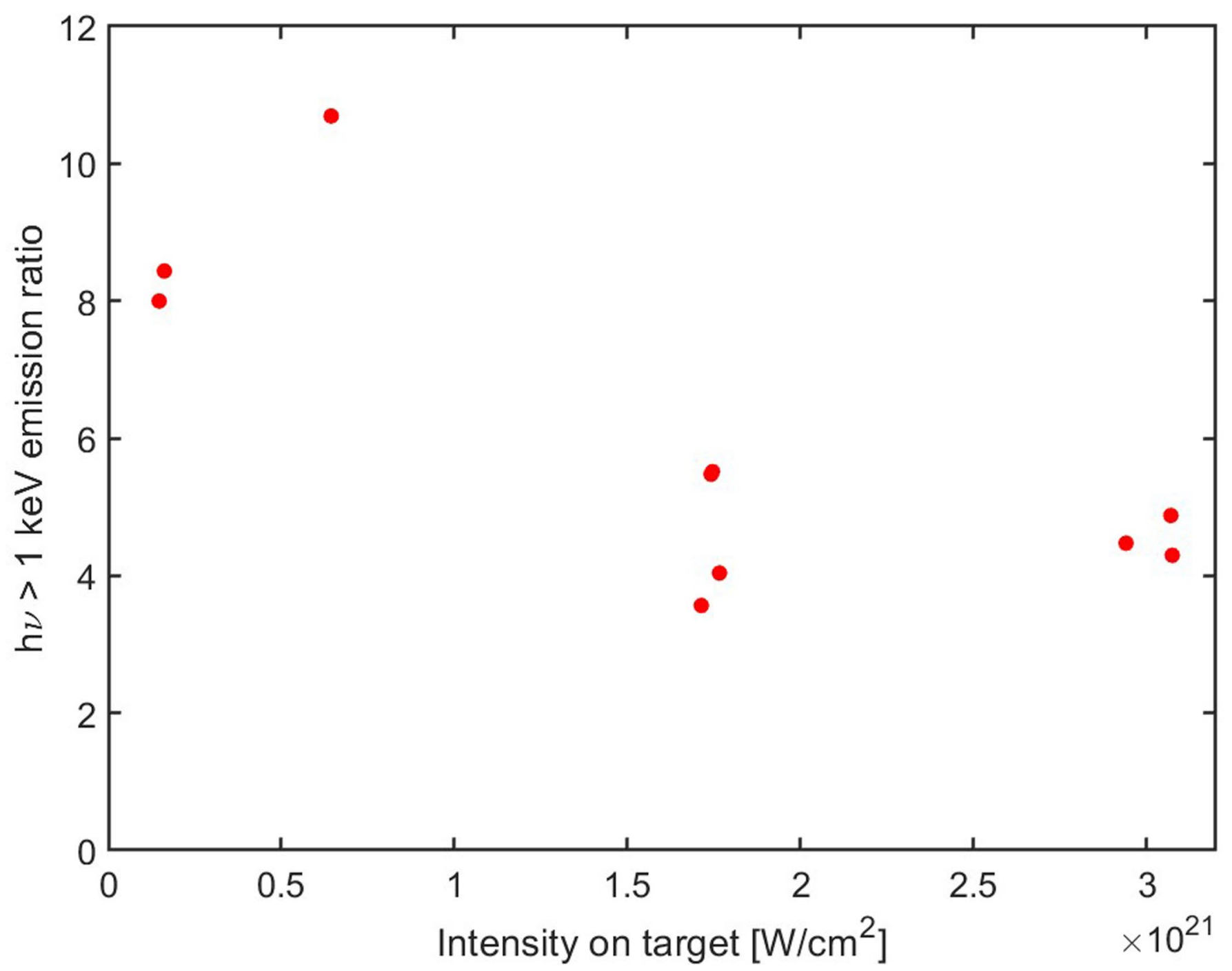

Extended Data Fig. 2 | Ratio of X-ray emission between nanowire and solid-density targets. Ratio of X-ray emission at photon energies $>1$ keV from an Au nanowire target (100 nm diameter nanowire array, $15 \%$ solid density) to Au solid foil target as a function of irradiation intensity. 


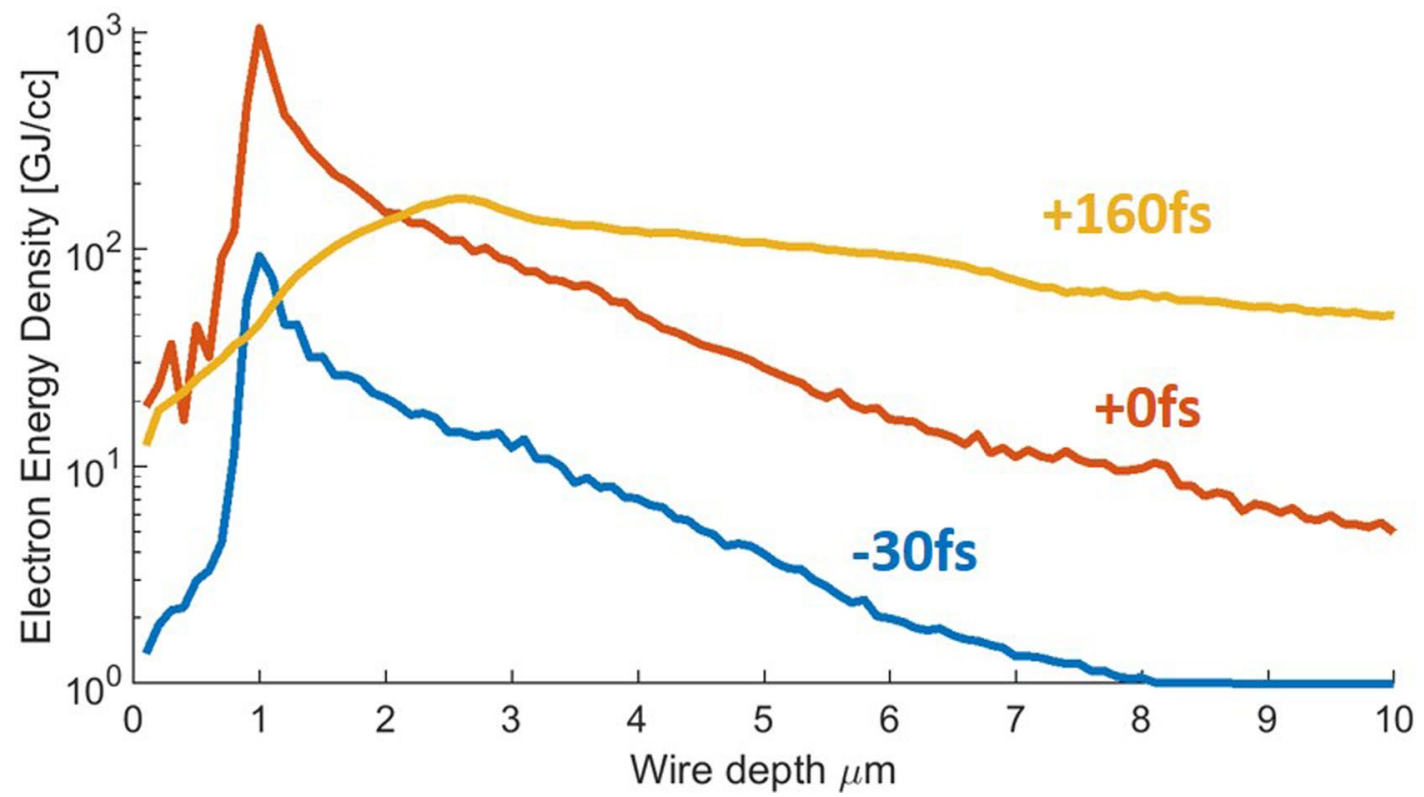

Extended Data Fig. 3 | Computed electron energy density. Computed electron energy density for an array of 100 nm diameter Au nanowire array (15\% solid density) plotted for three different times with respect to the peak of the laser pulse $(-30 \mathrm{fs}, 0 \mathrm{fs}$ and $+160 \mathrm{fs})$. Irradiation intensity $4 \times 10^{21} \mathrm{Wcm}^{-2}$. 


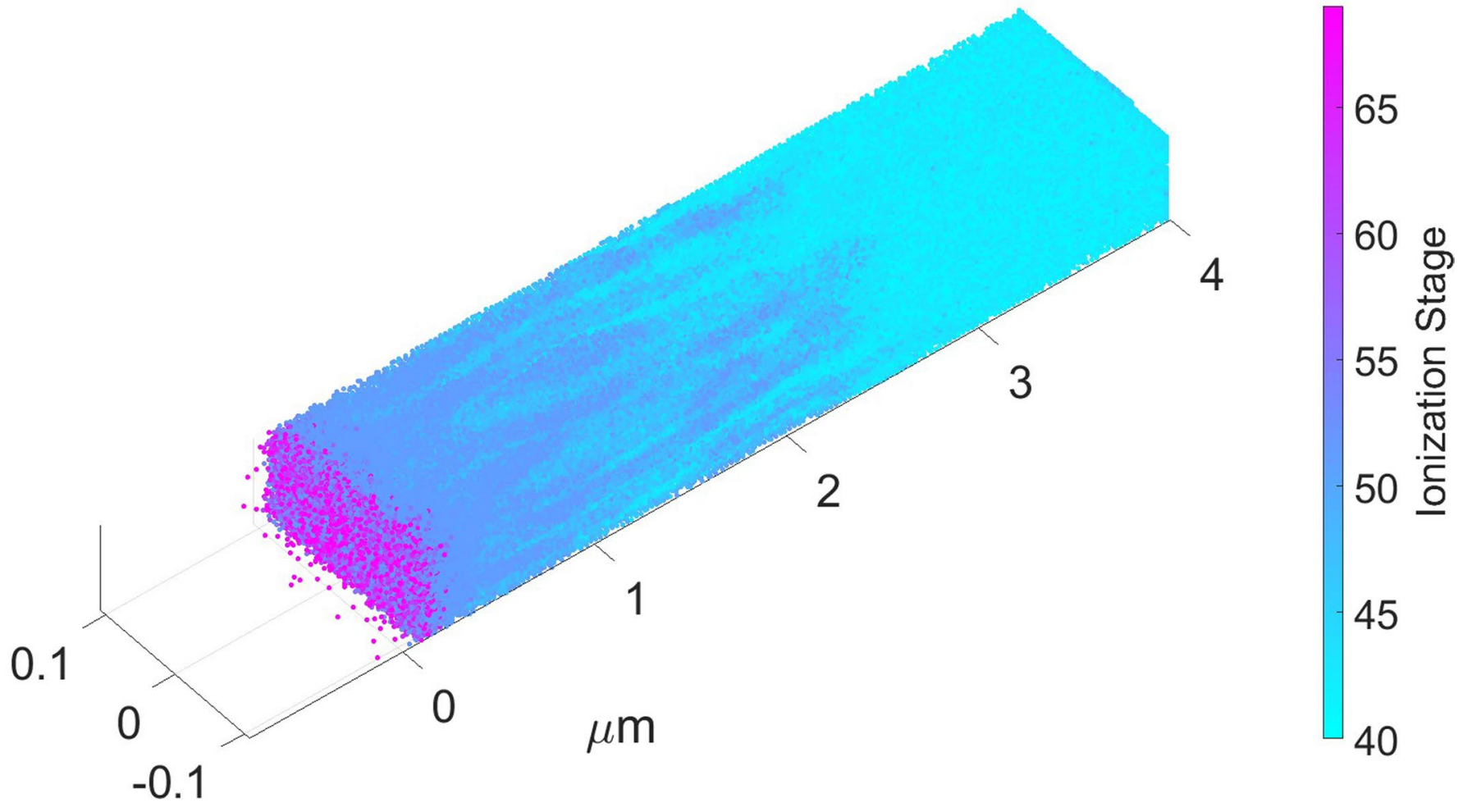

Extended Data Fig. 4 | High-resolution 3D particle in cell simulation of solid-density ionization. Predicted ionization stage for an infinite (periodically bounded in the transverse direction) solid Au flat target at $+30 \mathrm{fs}$ following the peak of the laser pulse. 


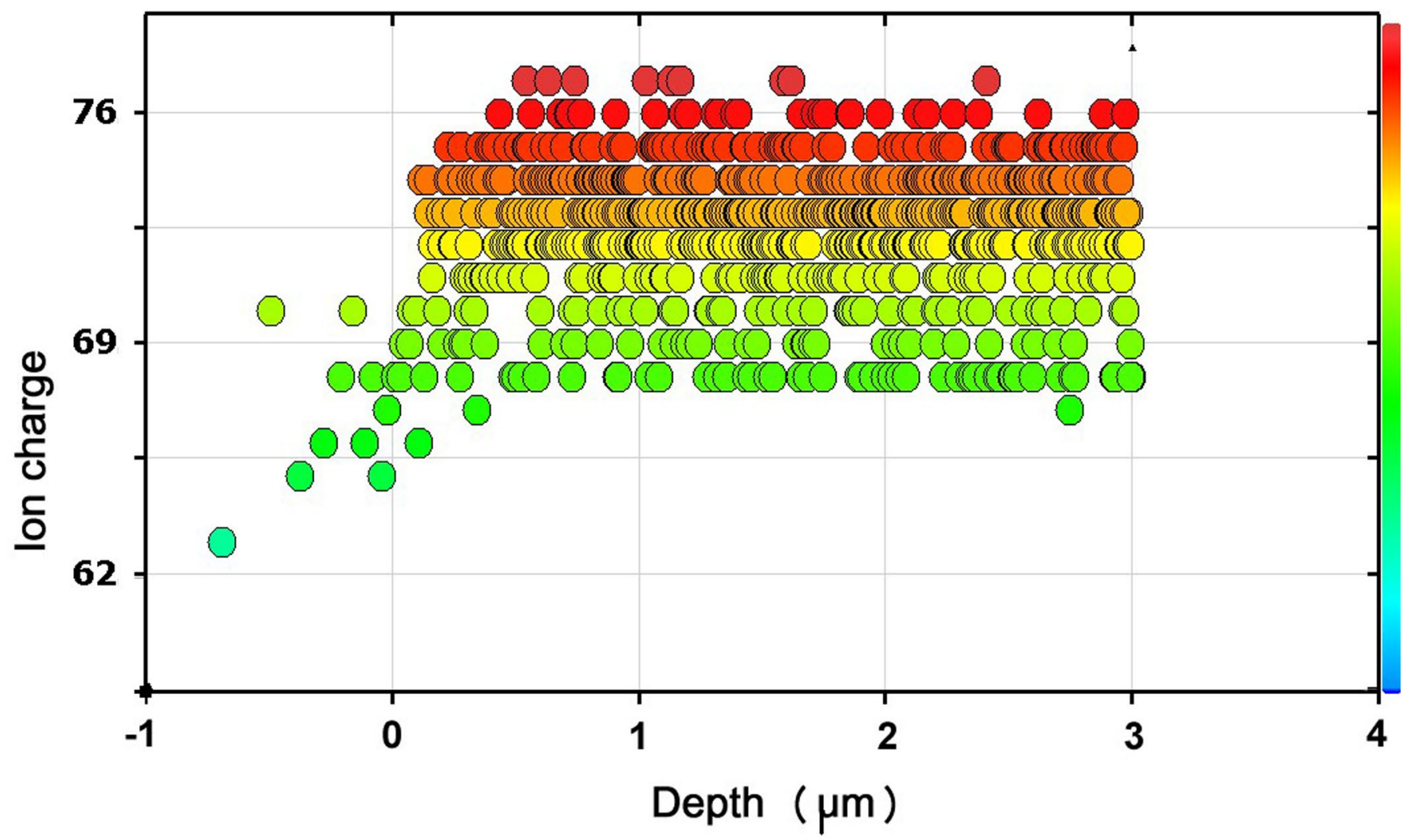

Extended Data Fig. 5 | Computed ionization for a 5 micron diameter laser spot. Computed ionization state as a function of target depth for a solid-density Au foil irradiated at an intensity of $4 \times 10^{21} \mathrm{Wcm}^{-2}$ with a $5 \mu \mathrm{m}$ FWHM diameter focal spot showing atoms ionized up to $\mathrm{He}^{-l i k e ~} \mathrm{Au}^{77+}$. 


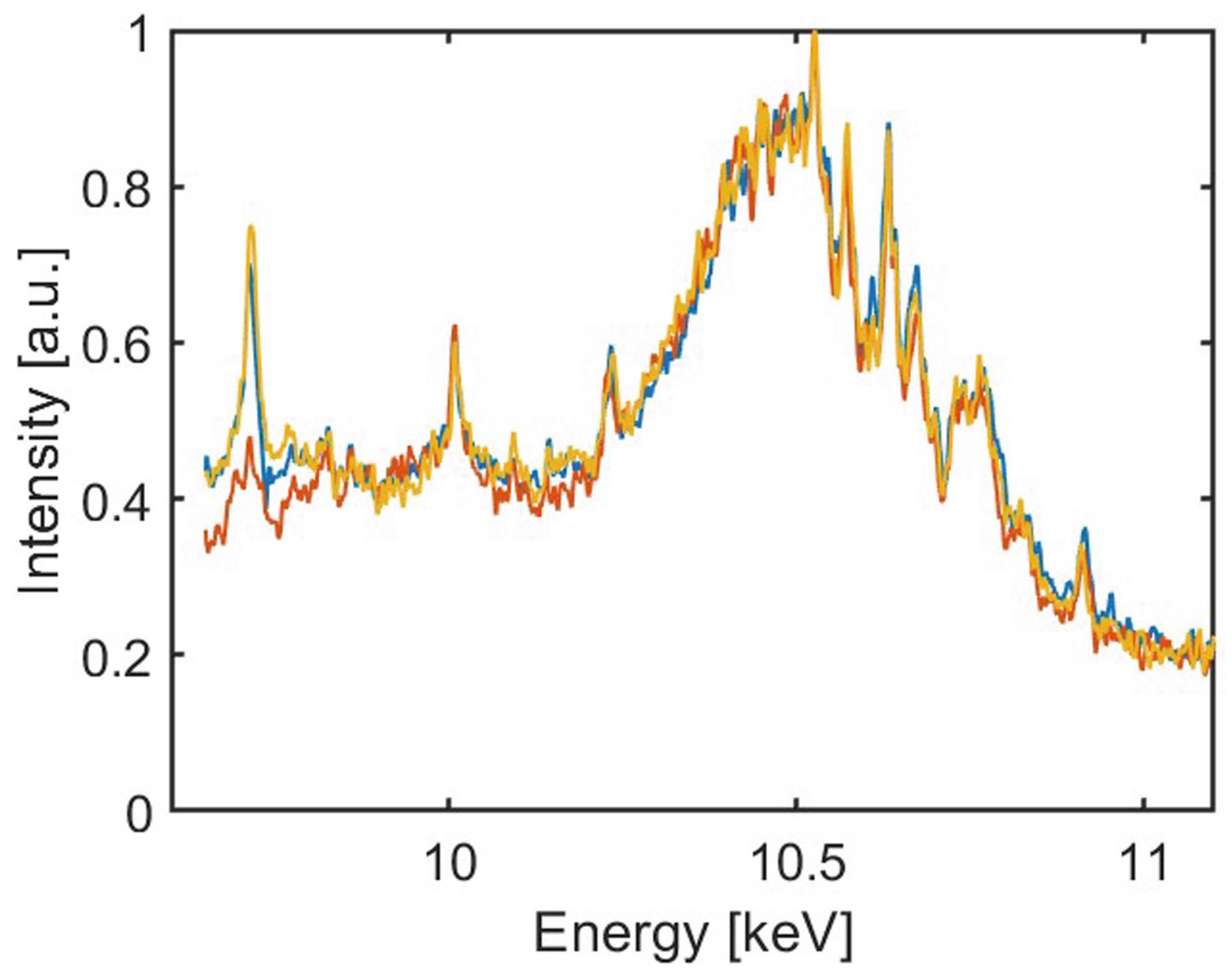

Extended Data Fig. 6 | Shot to shot reproducibility. Three consecutive single shot Au L shell spectra from an Au foil normalized by the Ne-like Au line emission showing good shot to shot reproducibility. 


\section{NATURE PHOTONICS}

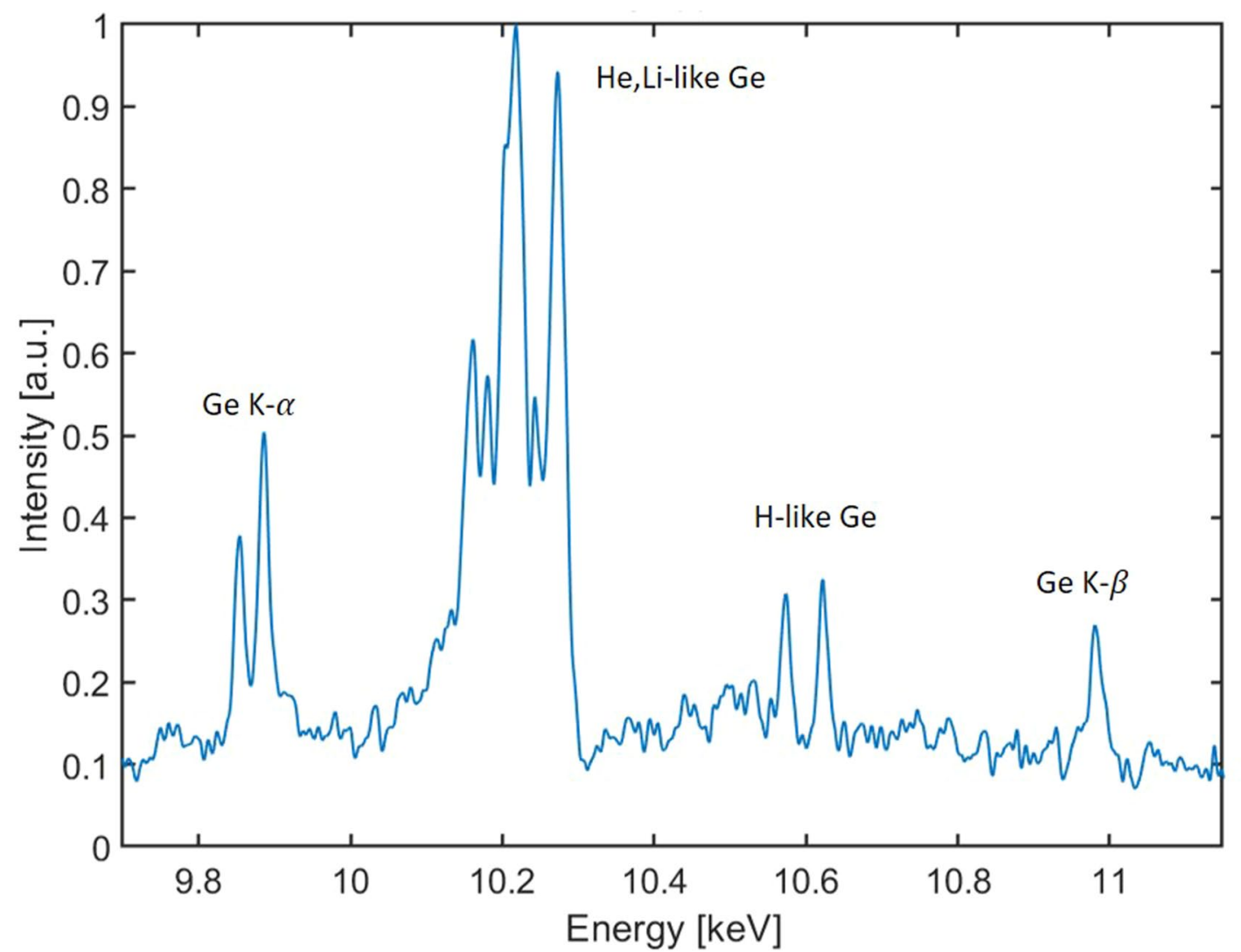

Extended Data Fig. 7 | Ge K-shell spectrum used to calibrate Au L-shell. K-shell spectrum of the Ge plasma used to calibrate the Von Hamos spectrometer in the 9.7-11.2 keV spectral range. The plasma was generated irradiating a $17 \mu \mathrm{m}$ thick Ge wafer at an intensity of $3 \times 10^{21} \mathrm{~W} \mathrm{~cm}^{-2}$. 